\title{
Vital sign sensing method based on EMD in terahertz band
}

\author{
Zhengwu $\mathrm{Xu}^{*}$ and Tong Liu
}

\begin{abstract}
Non-contact respiration and heartbeat rates detection could be applied to find survivors trapped in the disaster or the remote monitoring of the respiration and heartbeat of a patient. This study presents an improved algorithm that extracts the respiration and heartbeat rates of humans by utilizing the terahertz radar, which further lessens the effects of noise, suppresses the cross-term, and enhances the detection accuracy. A human target echo model for the terahertz radar is first presented. Combining the over-sampling method, low-pass filter, and Empirical Mode Decomposition improves the signal-to-noise ratio. The smoothed pseudo Wigner-Ville distribution time-frequency technique and the centroid of the spectrogram are used to estimate the instantaneous velocity of the target's cardiopulmonary motion. The down-sampling method is adopted to prevent serious distortion. Finally, a second time-frequency analysis is applied to the centroid curve to extract the respiration and heartbeat rates of the individual. Simulation results show that compared with the previously presented vital sign sensing method, the improved algorithm enhances the signal-to-noise ratio to $1 \mathrm{~dB}$ with a detection accuracy of $80 \%$. The improved algorithm is an effective approach for the detection of respiration and heartbeat signal in a complicated environment.
\end{abstract}

Keywords: Terahertz; Empirical Mode Decomposition; Smoothed pseudo Wigner-Ville distribution; Respiration and heartbeat rates detection; Performance analysis

\section{Introduction}

Respiration and heartbeat rates detection is required for health examinations, particularly with aging society [1], which is becoming an increasingly important issue. Traditional products and methods are of the contact type, that is, these approaches require contact with or attachment of some sensors to the patient's body. These techniques include electrocardiography (ECG), oximetry, respiration belt, and so on. Such methods are difficult to employ for infants and for severely scalded or burned patients. Microwave technology can be utilized for noncontact respiration and heartbeat rates detection, which is not confronted by the aforementioned problems [2-5]. This technology supports respiration and heartbeat rates detection in an out-of-hospital environment and facilitates remote monitoring in a hospital to improve user satisfaction. Meanwhile, the detection process is

\footnotetext{
* Correspondence: zwxu@uestc.edu.cn

School of Electronic Engineering, University of Electronic Science and Technology of China, Chengdu 611731, China
}

unnoticeable and avoids the user's respiration and heartbeat rates change caused by mood swing.

The terahertz $(\mathrm{THz})$ radar has unique advantages in the field of non-contract respiration and heartbeat rates detection. The $\mathrm{THz}$ wave has a shorter wavelength than other bands. The Doppler shift is inversely proportional to the wavelength. A shorter wavelength will generate a larger micro-Doppler frequency for the same micro-motion. The $\mathrm{THz}$ radar makes the features of the micro-Doppler more visible and can thus improve the detection accuracy [6]. The $\mathrm{THz}$ photon has minimal energy. The $\mathrm{THz}$ wave differs from X-ray in that it has no ionization effect and does not destroy the original structure of the material [7]. Thus, the $\mathrm{THz}$ wave will not damage the human body. Moreover, the $\mathrm{THz}$ wave can penetrate some non-metallic materials and can be reflected back from human targets. The terahertz radar can be used to detect respiration and heartbeat rates of survivors trapped in earthquakes and avalanches [8].

The Empirical Mode Decomposition (EMD) can filter the noise from ECG signals [9,10]. Basing from

\section{实}

(c) 2014 Xu and Liu; licensee Springer. This is an Open Access article distributed under the terms of the Creative Commons Attribution License (http://creativecommons.org/licenses/by/4.0), which permits unrestricted use, distribution, and reproduction in any medium, provided the original work is properly credited. 
EMD, we propose an improved respiration and heartbeat rates detection algorithm with $\mathrm{THz}$ radar that can further enhance the signal-to-noise ratio (SNR). This approach can effectively detect respiration and heartbeat signals in complicated environments with low SNR.

Section 2 introduces the heartbeat and respiration signal model. Section 3 introduces the Empirical Mode Decomposition and presents the steps of the improved respiration and heartbeat rates detection algorithm. Section 4 discusses the performance analysis of the improved respiration and heartbeat rates detection algorithm. Section 5 presents the conclusion.

\section{Heartbeat and respiration signal model}

For the $\mathrm{THz}$ continuous wave radar, we assume that the transmitter and receiver are located at the origin. The signal transmitted at time $t$ can be defined as

$$
S_{\mathrm{T}}(t)=e^{j 2 \pi f_{\mathrm{c}} t}
$$

where $f_{\mathrm{c}}$ is the frequency of the transmitted signal. In practice, $f_{\mathrm{c}}$ is $240 \mathrm{GHz}$. The signal received at time $t$ is

$$
S_{\mathrm{R}}(t)=e^{j 2 \pi f_{c}\left(t-\frac{2 R(t)}{c}\right)}
$$

where $c$ is the speed of light, and the delay of the radar echo is $2 R(t) / c$. This assumption is known as the startstop approximation. The speed of light is significantly faster than that of the subject body. Thus, the correctness of the assumption becomes more accurate to a higher degree.

Once the radar signal bounces off the subject, its energy is collected by the receiver. The signal is then demodulated with a local oscillator. The modulated received signal is expressed as

$$
S_{\mathrm{MR}}(t)=S_{\mathrm{R}}(t) \cdot e^{-j 2 \pi f_{\mathrm{c}} t}=e^{-j 2 \pi\left(2 \lambda^{-1} R(t)\right)}
$$

where $\lambda=c / f_{\mathrm{c}}$ is the wavelength of the radar wave.

Some harmonic components also exist aside from the main frequencies referred to by the ECG data. The periodic motion of respiration can be considered as a sinusoidal model. The analysis is simplified by assuming that the human body is stationary, that is, the speed of the target is zero. The human target model can be established as

$$
R(t)=R_{0}+r_{1} \sin \left(2 \pi f_{1} t\right)+r_{2} \delta\left(f_{2} t-\tau\right)
$$

where $R_{0}$ is the overall fixed distance from the person to the radar; $r_{1}$ and $r_{2}$ are the displacements of respiration and heartbeat, respectively; $f_{1}$ and $f_{2}$ is the frequencies of respiration and heartbeat, respectively;
Table 1 The respiration and heartbeat signal model parameters

\begin{tabular}{lc}
\hline Parameter & Value \\
\hline Carrier frequency $f_{c}$ & $240 \mathrm{GHz}$ \\
Fixed distance $R_{0}$ & $50 \mathrm{~m}$ \\
Respiration displacement $r_{1}$ & $5 \mathrm{~mm}$ \\
Heartbeat displacement $r_{2}$ & $0.8 \mathrm{~mm}$ \\
Respiration rate $f_{1}$ & $0.23 \mathrm{~Hz}$ \\
Heartbeat rate $f_{2}$ & $1 \mathrm{~Hz}$ \\
Heartbeat radius $r$ & $0.25 \mathrm{~m}$ \\
Heart rate shift $\tau$ & 0 \\
Time $t$ & $30 \mathrm{~s}$ \\
SNR & $-13 \mathrm{~dB}$ \\
\hline
\end{tabular}

and $\tau$ is the heart rate shift. Meanwhile, $\delta$ is defined as

$$
\delta(t)=\frac{1}{1-2 a}\left(\left|t-\frac{1}{2}-\lfloor t\rfloor\right|-a+|| t-\frac{1}{2}-\lfloor t\rfloor|-a|\right)
$$

for $a=1 / 2-r \cdot f_{2}$, where $r$ is the heartbeat radius.

The parameters are shown in Table 1, and the real part of noised $S_{\mathrm{MR}}(t)$ is shown in Figure 1 .

\section{Improved detection algorithms}

Combining the EMD and the previously reported respiration and heartbeat rates detection algorithm [11] significantly improves the latter.

\subsection{EMD method}

EMD, a new adaptive and effective data decomposition method aiming at non-linear and non-stationary data, was presented by NE Huang in 1998 [12]. The decomposition process involves the sifting of the intrinsic

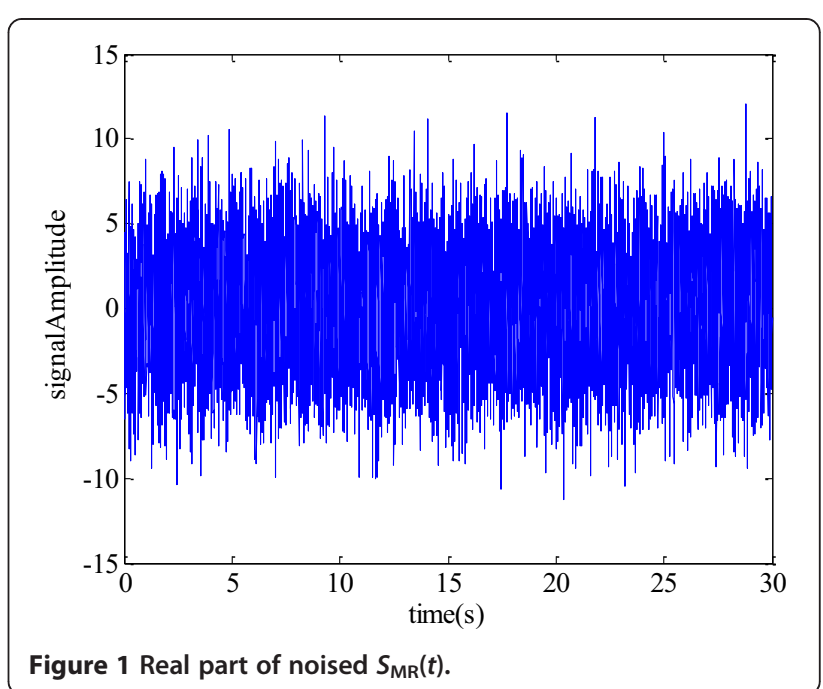


oscillatory modes with their characteristic time scales in the signal, which is fully driven by the data. Any given signal can be decomposed into a series of intrinsic mode functions (IMFs). Every IMF confirms a certain frequency band of the signal $s(t)$. The IMF sifted earlier represents a higher frequency band than that sifted later. The IMF should satisfy two conditions: (1) the number of local extrema and that of zero crossings can differ by one at most for the whole data, and (2) the mean value of the envelope defined by the local maxima and that defined by the local minima should be zero at any point [13]. These conditions indicate that the intrinsic mode function is a single-component signal. That is, any given multi-component signal $s(t)$ can be decomposed into several single-component signals with the EMD approach.

For any given signal $s(t)$, the EMD process is as follows:

Step 1: All the local extrema of $s(t)$ are located. The local maxima and minima of $s(t)$ are interpolated by cubic spline to compute for the upper envelope $s_{\max }(t)$ and the lower envelope $s_{\min }(t)$ of $s(t) . m(t)$ is the envelope mean of $s_{\max }(t)$ and $s_{\min }(t)$, whereas $h(t)$ is the difference between $s(t)$ and $m(t)$.

$$
\begin{aligned}
& m(t)=\left[s_{\max }(t)+s_{\min }(t)\right] / 2 \\
& h(t)=s(t)-m(t)
\end{aligned}
$$

Step 2: $h(t)$ is considered as $s(t)$. Step 1 is repeated until $h(t)$ satisfies the intrinsic mode function conditions. We set $\operatorname{imf}_{1}(t)=h(t)$, and $\operatorname{imf}_{1}(t)$ is an IMF. The residue is given by

$$
r_{1}(t)=s(t)-\mathrm{imf}_{1}(t)
$$

Step 3: $r_{1}(t)$ is regarded as $s(t)$. Step 1 and step 2 are repeated to identify other $\operatorname{IMFs~}_{\operatorname{imf}_{2}}(t), \operatorname{imf}_{3}(t) \ldots$, and residuals $r_{2}(t), r_{3}(t) \ldots$, in sequence. If the amplitude of $\operatorname{imf}_{N}(t)$ is small enough or the number of extrema of $r_{N}(t)$ is less than a predetermined number, the whole decomposition process stops.

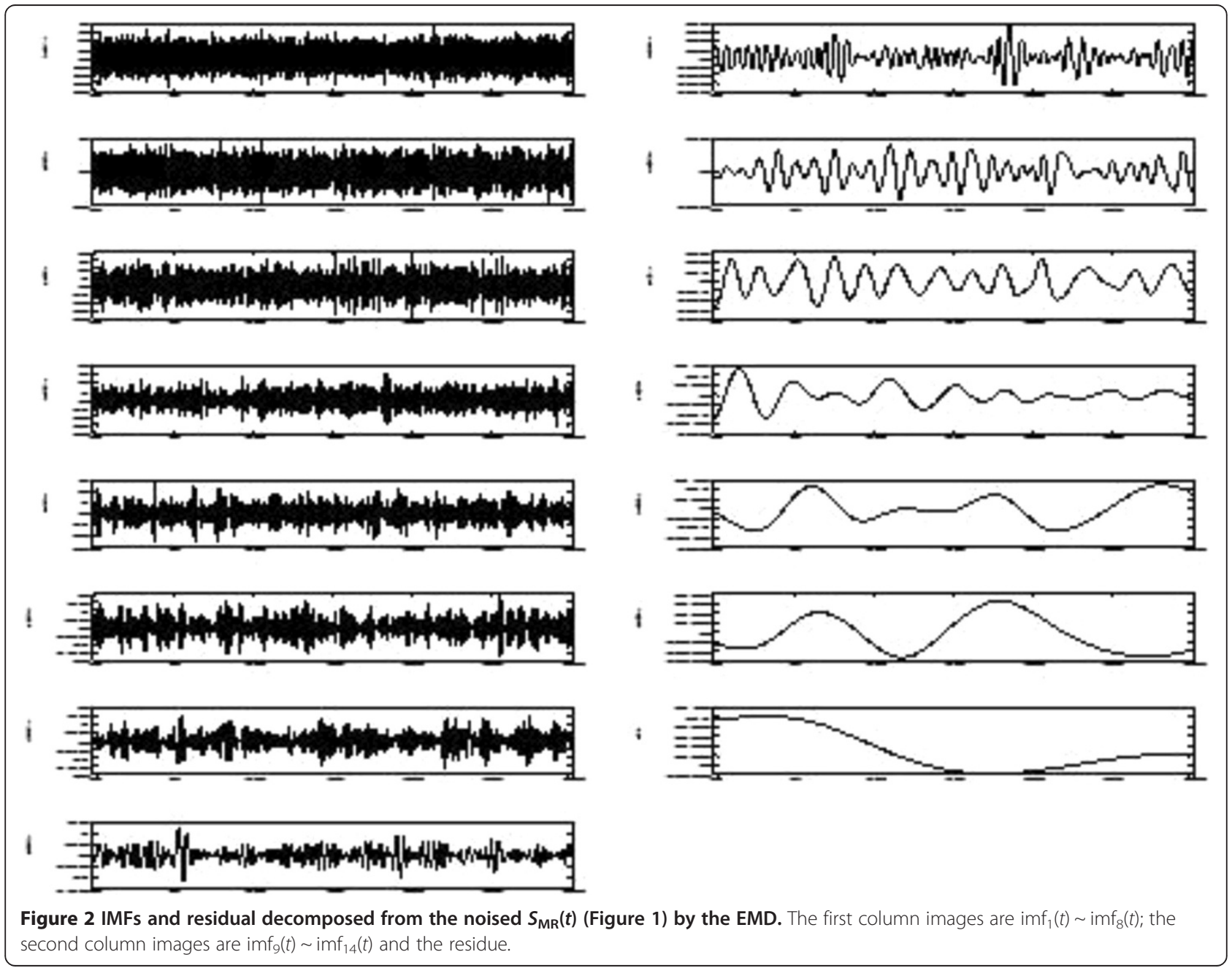




\section{Echo $\rightarrow$ EMD $\rightarrow$ SPWVD $\rightarrow$ Estimation of $R^{\prime}(t) \rightarrow$ SPWVD $\rightarrow$ Separate the frequency}

Figure 3 Improved initial algorithm flowchart.

With the EMD approach, $s(t)$ is represented as linear superposition of IMFs $\operatorname{imf}_{i}(t)$ and the residue $r_{N}(t)$.

$$
s(t)=\sum_{i=1}^{N} \operatorname{imf}_{i}(t)+r_{N}(t)
$$

Figure 2 shows the decomposition of the noised $S_{\mathrm{MR}}(t)$ (Figure 1) into IMFs and the residue by the EMD.

The IMF is not a single-component signal but contains a certain frequency band of the noised $S_{\mathrm{MR}}(t)$ (Figure 1) because of the interpolation error and end effect.

\subsection{The improved respiration and heartbeat rates detection algorithm}

A respiration and heartbeat rates detection algorithm was previously presented [11]. For the continuous wave after demodulation, the Doppler frequency is given by

$$
f_{\mathrm{D}}=\frac{d}{d t}\left(2 \lambda^{-1} R(t)\right)=\frac{2 \dot{R(}(t)}{\lambda}
$$

where $\lambda$ is the signal wavelength. Thus, the Doppler shift in the received radar signal is proportional to the velocity of the target $\dot{R}(t)$.

The smoothed pseudo Wigner-Ville distribution (SPWVD) time-frequency technique is applied to the respiration and heartbeat signal to measure the Doppler frequency of the target. The centroid of the spectrogram estimates the instantaneous velocity of target's cardiopulmonary motion $\dot{R}(t)$. Finally, the centroid curve undergoes a second timefrequency analysis to extract the respiration and heartbeat rates of the individual.

The maximum of the Doppler frequency is

$$
\begin{aligned}
f_{\mathrm{D} \max } & =\max \left\{\frac{d}{d t}\left(2 \lambda^{-1} R(t)\right)\right\} \\
& =\max \left\{\frac{d}{d t}\left(2 \lambda^{-1}\left(R_{0}+r_{1} \sin \left(2 \pi f_{1} t\right)+r_{2} \delta\left(f_{2} t-\tau\right)\right)\right)\right\} \\
& =2 \lambda^{-1} \max \left\{2 \pi f_{1} r_{1} \cos \left(2 \pi f_{1} t\right)\right\}+2 \lambda^{-1} \max \left\{r_{2} \frac{d}{d t}\left(\delta\left(f_{2} t-\tau\right)\right)\right\} \\
& =2 \lambda^{-1} \max \left\{2 \pi f_{1} r_{1}\right\}+2 \lambda^{-1} \max \left\{\frac{r_{2}}{r}\right\}
\end{aligned}
$$

where $\lambda$ is fixed. In practice, the ceiling limit values of $f_{1}$, $r_{1}$, and $r_{2}$ exist, the lower limit value of $r$ exists. Thus, the maximum of the Doppler frequency $f_{\mathrm{D} \text { max }}$ can almost be confirmed.

An IMF confirms a certain frequency band of the respiration and heartbeat signal. Utilizing $f_{\mathrm{D} \text { max }}$ as threshold, the IMFs constituted with the required frequency components can be separated and the IMFs constituted with noise can be eliminated. The noise from the noised respiration and heartbeat signal can be further lessened by the EMD method. The improved initial algorithm processes are shown in Figure 3.

Given that the noised respiration and heartbeat signal is a multi-component signal, the SPWVD based on the EMD is applied to the respiration and heartbeat signal to suppress the cross-term, and the centroid curve extraction method is employed to approximate $\dot{R}(t)$. The improved detailed flowchart is shown in Figure 4.

The over-sampling method is employed in the echo signal as pre-treatment. When the signal passes through

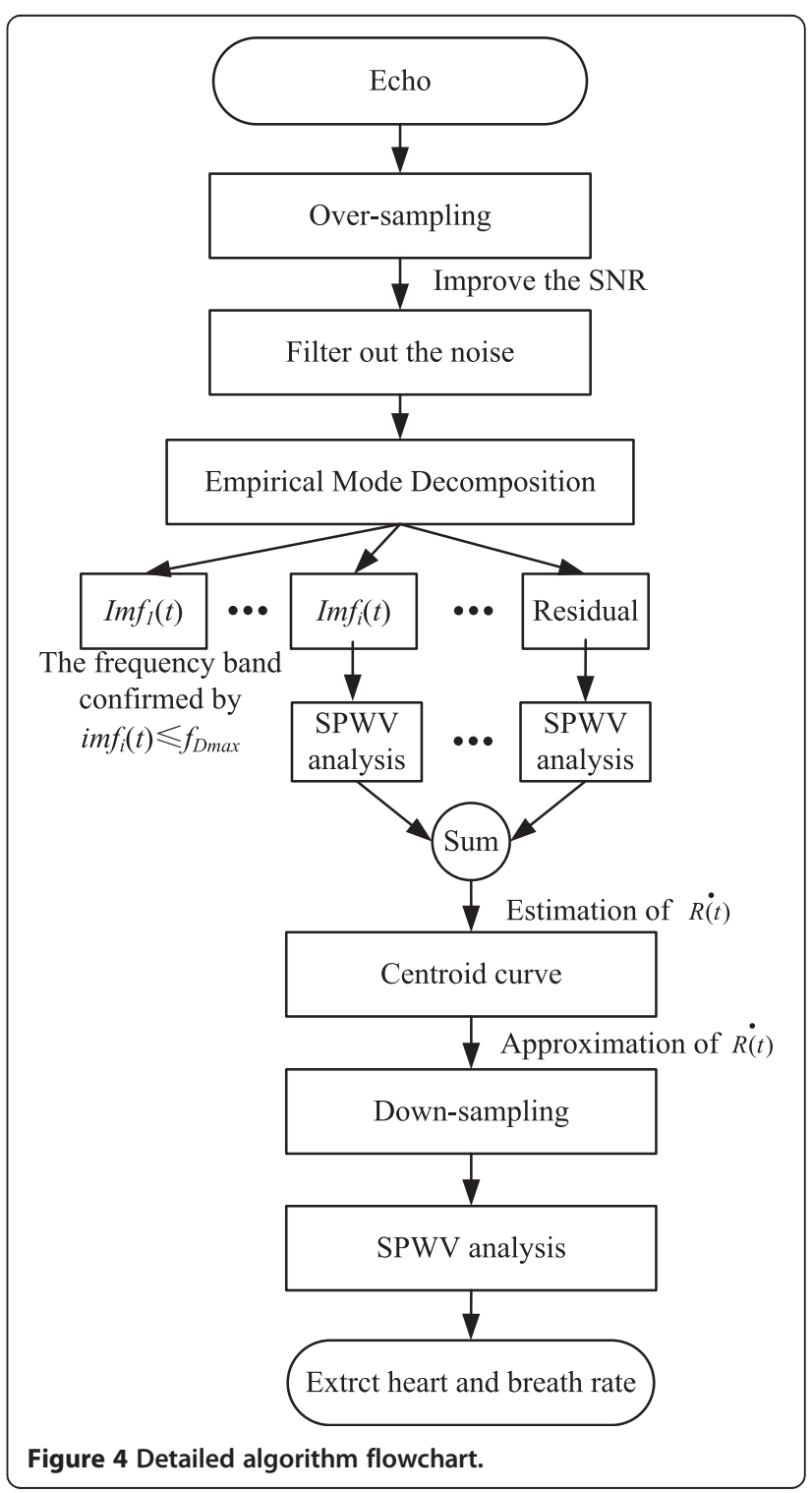


Table 2 Simulation parameters

\begin{tabular}{lc}
\hline Parameter & Value \\
\hline Carrier frequency $f_{\mathrm{c}}$ & $240 \mathrm{GHz}$ \\
Time $t$ & $30 \mathrm{~s}$ \\
Sampling rate $1 f_{\mathrm{O}}$ & $500 \mathrm{~Hz}$ \\
Sampling rate $2 f_{\mathrm{d}}$ & $10 \mathrm{~Hz}$ \\
Window $1 \mathrm{~h}=$ hamming & 127 \\
Window $2 \mathrm{~g}=$ hamming & 127 \\
Frequency points $N$ & 512 \\
Signal-to-noise ratio SNR & $-13 \mathrm{~dB}$ \\
\hline
\end{tabular}

the first time-frequency analysis, the sampling frequency is larger than the maximal frequency component in the signal. Noise components can be filtered out by a lowpass filter, which can help improve the SNR of the initial analysis of the weak signal and lessen the effects of noise on the signal band.

The EMD method is employed to filter noise further. Combined with the SPWVD time-frequency technique, the instantaneous Doppler frequency of the target is obtained. Utilizing $f_{\mathrm{D}}$ max as threshold, the IMFs constituted with noise are eliminated with the EMD approach. The retained IMFs and residue are respectively

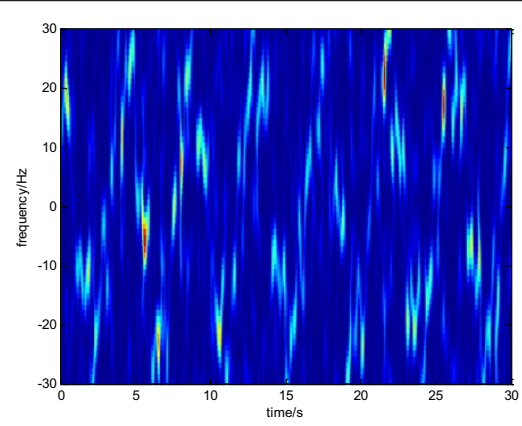

(a)

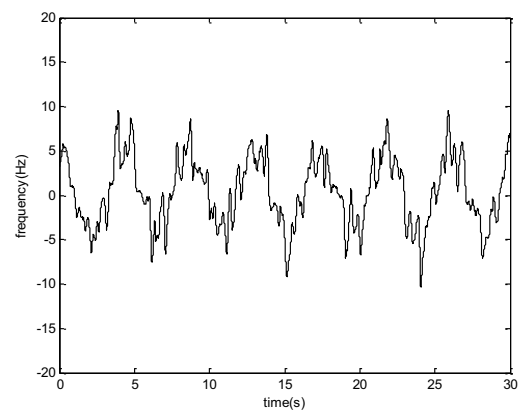

(c)

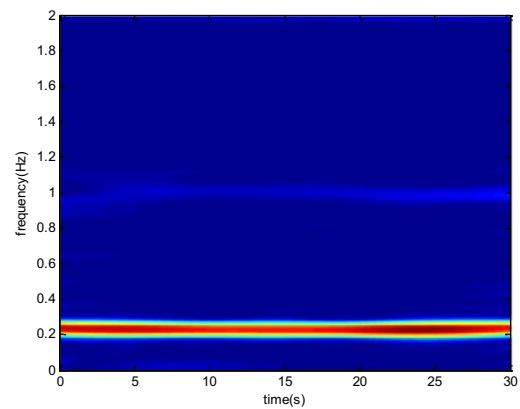

(e)

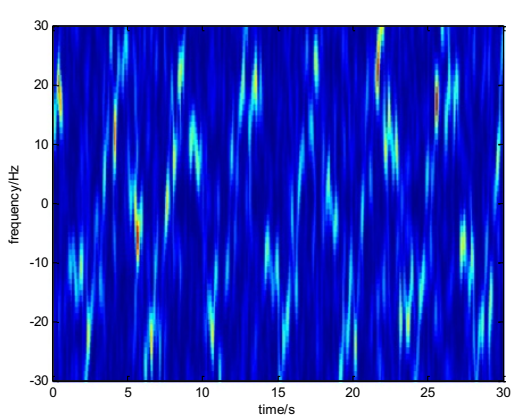

(b)

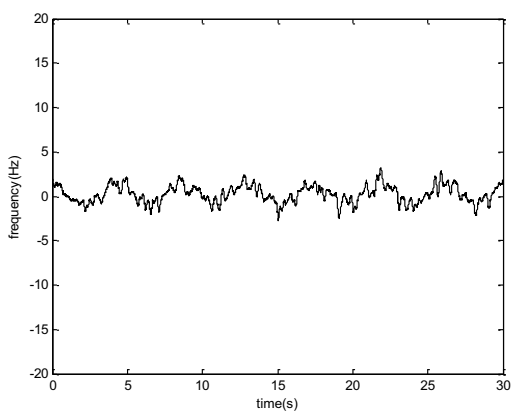

(d)

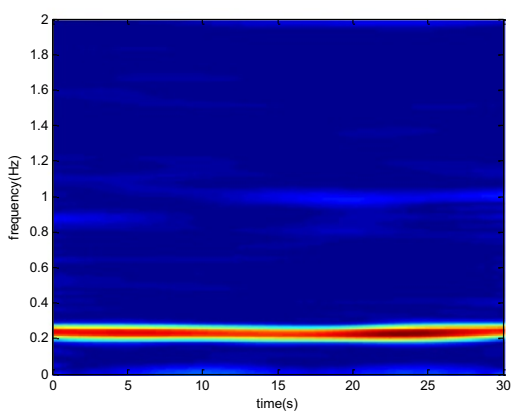

(f)

Figure 5 Comparison of previously presented detection algorithm and improved detection algorithm. (a) SPWVD of echo (the improved algorithm). (b) SPWVD of echo. (c) Centroid (the improved algorithm). (d) Centroid. (e) SPWVD of centroid (the improved algorithm). (f) SPWVD of centroid. 
calculated by using SPWVD. The time-frequency result of the reconstituted respiration and heartbeat signal EMD _ SPWV $(t, f)$ is the sum of the time-frequency results of the retained IMFs and the residue.

$$
\text { EMD_SPWV }(t, f)=\sum_{i=L}^{N} \operatorname{SPWV}_{\operatorname{imf}_{i}}(t, f)+\operatorname{SPWV}_{r_{N}}(t, f)
$$

where $\operatorname{imf}_{1}(t) \cdots \operatorname{imf}_{L-1}(t)$ are the eliminated IMFs, the frequency band confirmed by $\operatorname{imf}_{L}(t)$ includes $f_{\mathrm{D} \text { max }}$, $\operatorname{SPWV}_{\operatorname{imf}_{i}}(t, f)$ is the time-frequency result of $\operatorname{imf}_{i}(t)$, and $\operatorname{SPWV}_{r_{N}}(t, f)$ is the time-frequency result of the residue $r_{\mathrm{N}}(t)$.

Numerous frequency components exist at any moment in the time-frequency diagram. The estimated $\dot{R}(t)$ cannot be obtained through a simple time-frequency analysis. Spectral centroid method can be used to estimate $\dot{R}(t)$ The weighted average of the spectrogram for each time $t$ and the pair of $(t, f)$ can be identified to determine the centroid of the spectrogram. The formula is as follows:

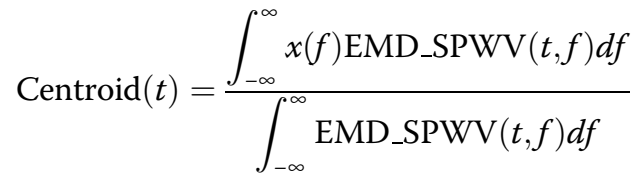

where $x(f)$ is the weighting function of the frequency, and the default value is $f$ for simplicity.

Before the second time-frequency analysis is executed, we extracted the centroid curve, which is close to the speed signal of the human body to a certain extent. Down-sampling is adopted to obtain a sampling frequency down to $10 \mathrm{~Hz}$, which also satisfies the Nyquist sampling theorem. If the sampling frequency of the second time-frequency analysis does not undergo downsampling, the final time-frequency diagram will exhibit serious distortion.

In the second time-frequency analysis of the centroid curve, the respiration and heartbeat frequencies become separated.

The parameters in Table 2 are utilized for simulation. A comparison of the previously presented detection algorithm and the improved detection algorithm is shown in Figure 5.

The time-frequency diagram of Figure $5 \mathrm{e}$ is purer than that of Figure $5 \mathrm{f}$. The improved algorithm enhances the SNR and suppresses the cross-term.

\section{Analysis of performance}

The width of the high spectral energy density areas is noticeable. The exact frequency cannot be determined. To extract the respiration and heartbeat frequencies, the

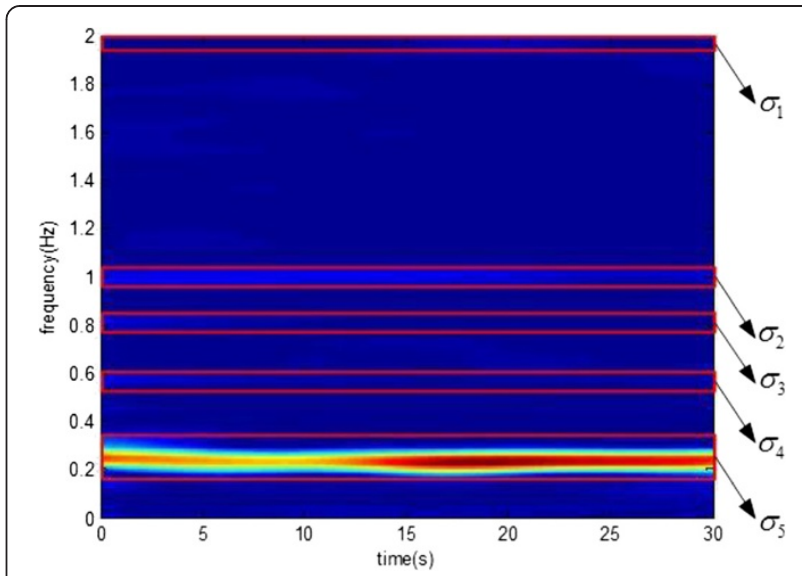

Figure 6 High spectral energy density areas.

following solution is required: the spectral energy density of the time-frequency diagram is normalized; the appropriate threshold is selected, and the high spectral energy density areas are sifted out as Figure 6 illustrated; for each frequency $f$ and the pair of $(t, f)$ in each high spectral energy density area, the maximal average of spectral energy density $W_{\sigma_{i}}$ and its corresponding frequency $f_{i}$ are identified as

$$
\begin{gathered}
f_{i}=\max _{f \in \sigma_{i}} \frac{1}{T_{\text {dur }}} \int_{-\infty}^{+\infty} \operatorname{SPWV}(t, f) d t \\
W_{\sigma_{i}}=\frac{1}{T_{\text {dur }}} \int_{-\infty}^{+\infty} \operatorname{SPWV}\left(t, f_{i}\right) d t
\end{gathered}
$$

where $T_{\text {dur }}$ is the signal observation time, and $\operatorname{SPWV}(t, f)$ is the normalized spectral energy density after the second time-frequency analysis. The $f_{i}$ corresponding to the largest $W_{\sigma_{i}}$ is the respiration frequency, whereas $f_{i}$ corresponding to the second largest $W_{\sigma_{i}}$ is the heartbeat

Table 3 High spectral energy density areas and their corresponding frequencies in Figure 6

\begin{tabular}{lcc}
\hline $\begin{array}{l}\text { High spectral } \\
\text { energy density } \\
\text { area } \boldsymbol{\sigma}_{\boldsymbol{i}}\end{array}$ & $\begin{array}{c}\text { Corresponding } \\
\text { frequency } \boldsymbol{f}_{\boldsymbol{i}}(\mathrm{Hz})\end{array}$ & $\begin{array}{c}\text { Average of normalized } \\
\text { spectral energy } \\
\text { density } \boldsymbol{W}_{\boldsymbol{\sigma}_{\boldsymbol{i}}}\end{array}$ \\
\hline$\sigma_{1}$ & 1.981 & 0.0078 \\
$\sigma_{2}$ & 1.003 & 0.0597 \\
$\sigma_{3}$ & 0.807 & 0.0132 \\
$\sigma_{4}$ & 0.592 & 0.0197 \\
$\sigma_{5}$ & 0.230 & 0.8387 \\
\hline
\end{tabular}


frequency. Table 3 provides the extract resolution of Figure 6.

As shown in Table 3, the respiration and heartbeat frequencies are 0.230 and $1.003 \mathrm{~Hz}$, respectively.

The threshold $f_{\mathrm{D} \text { max }}$ will affect the choice of IMFs. As $f_{\mathrm{D} \text { max }}$ approaches the authentic maximum of the Doppler frequency, the detection result becomes more ideal, as shown in Figure 7.

The frequency band confirmed by $\mathrm{imf}_{4}$ is close to the practical maximum of the Doppler frequency, and the detection result shown in Figure 7c is better.

According to the simulation parameters shown in Table 1, the authentic maximum of Doppler frequency is about $17 \mathrm{~Hz}$. In order to avoid the loss of the Doppler frequency, the value of $f_{\mathrm{D}}$ max is appropriately larger than $17 \mathrm{~Hz}$. Employing Equations 14 and 15, the accurate respiration and heartbeat frequencies can be extracted. When the respiration and heartbeat frequencies are 0.23 and $1.00 \mathrm{~Hz}$, respectively, the detection is successful; otherwise, the detection is failed. The performance curves of the improved algorithm based on the EMD and the previously reported algorithm are shown in Figure 8.

Compared with the previously presented vital sign sensing method, the improved algorithm enhances the SNR to $1 \mathrm{~dB}$ with a detection accuracy of $80 \%$.

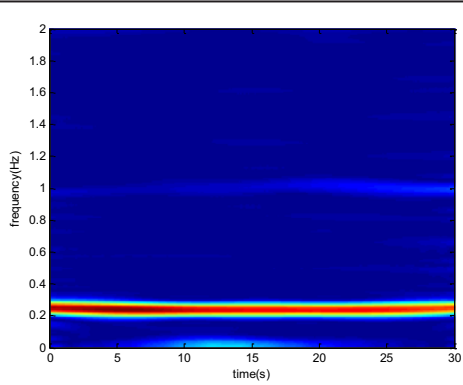

(a)

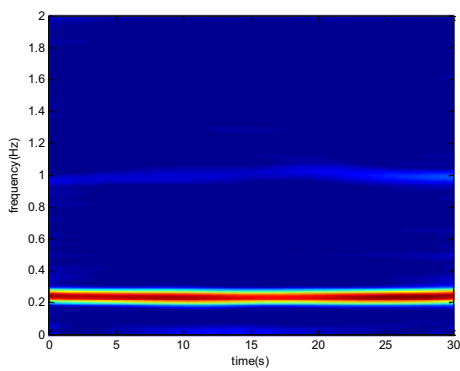

(c)

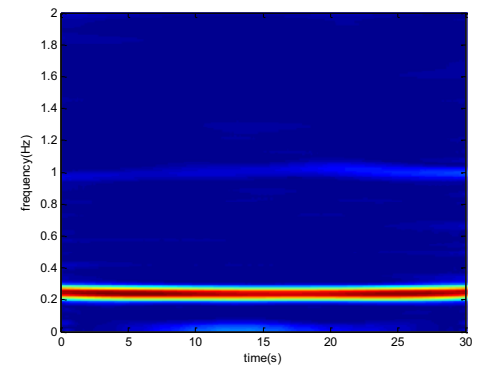

(b)

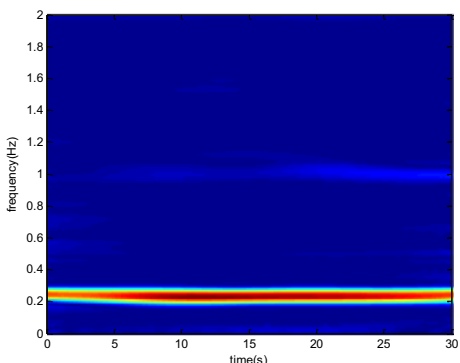

(d)

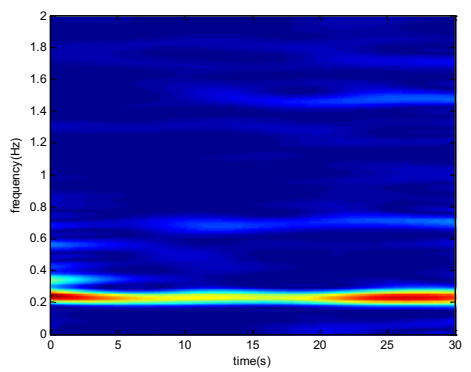

(e)

Figure 7 Choice of IMF effect on the detection result. (a) Detection result after elimination of the first IMF. (b) Detection result after elimination of the first two IMFs. (c) Detection result after elimination of the first three IMFs. (d) Detection result after elimination of the first four IMFs. (e) Detection result after elimination of the first five IMFs. 


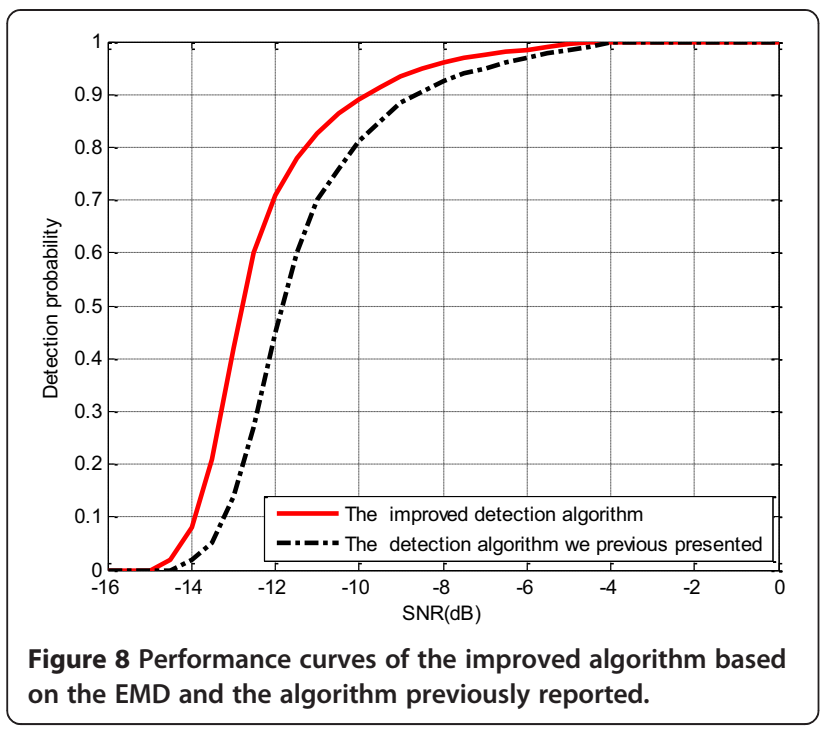

\section{Conclusions}

In this paper, a vital sign sensing method based on the EMD in $\mathrm{THz}$ band is proposed. The over-sampling method and low-pass filter sift out the noise components. The EMD further improves the SNR, and the downsampling method prevents serious distortion. Combining the SPWVD and the centroid curve method facilitates the extraction of the respiration and heartbeat rates with low SNR. Meanwhile, the detection performance of this method is analyzed. The improved respiration and heartbeat rates detection method is an effective approach for the analysis of the $\mathrm{THz}$ radar signal for vital sign sensing in a complicated environment.

\section{Competing interests}

The authors declare that they have no competing interests.

\section{Acknowledgements}

This work is supported by the National Natural Science Foundation of China under Project 61371048, the Fundamental Research Funds for the Central Universities under Project ZYGX2012J029, and the Fundamental Research Funds for the Central Universities under Project ZYGX2012Z001.

Received: 28 February 2014 Accepted: 6 May 2014

Published: 22 May 2014

\section{References}

1. RN Anderson, A method for constructing complete annual U.S. life tables. National Center for Health Statistics. Vital. and Health. Stat. 2(129), 1-28 (1999)

2. PH Siegel, Terahertz technology in biology and medicine. IEEE Trans. Microw. Theory Tech. 52(10), 2438-2447 (2004)

3. EF Greneker, Radar sensing of heartbeat and respiration at a distance with applications of the technology. Radar. 97, 150-154 (1997)

4. F Michahelles, W Ramon, S Bernt, Less contact: heart-rate detection without even touching the user, in Proceedings of the Eighth International Symposium on Wearable Computers, ed. by. Arlington, VA, USA, 31 October to 3 November 2004, pp 4-7

5. S-G Kim, G-H Yoon, J-G Yook, Sensitivity enhanced noncontact vital signal sensor using oscillation frequency deviation, in Proceedings of the Asia-Pacific Microwave Conference, ed. by (Melbourne, Victoria, Australia). 5-8 December 2011, pp 1586-1589
6. $L$ Jin, $P$ Yiming, Micro-Doppler signature feature analysis in terahertz band. J. Infrared Millimeter Waves 31(3), 319-328 (2010)

7. M Nagel, P Haring Bolivar, M Brucherseifer, H Kurz, A Bosserhoff, R Büttner, Intergrated THz technology for label-free genetic diagnostics. Appl. Phys. Lett. 80(1), 154-156 (2002)

8. YF Chen, $H$ Yong, Z Jianping, A Norman, Microwave life detection systems for searching human subjects under earthquake rubble or behind barrier. IEEE Trans. Biomed. Eng. 47(1), 105-114 (2000)

9. J Lee, DD McManus, S Merchant, KH Chon, Automatic motion and noise artifact detection in Holter ECG data using empirical mode decomposition and statistical approaches. IEEE Trans. Biomed. Eng. 59(6), 1499-1506 (2012)

10. A Dliou, R Lalif, M Laaboubi, F Maoulainine, S Elouaham, Noised abnormal ECG signal analysis by combining EMD and Choi-Williams techniques, in International Conference on Complex Systems, ed. by (Agadir, Morocco). 5-6 November 2012, pp 1-5

11. $X$ Zhengwu, $W$ Yuanjie, $L$ Xiaoqian, Time-frequency analysis of terahertz radar signal for vital signs sensing based on radar sensor. Int. J. of Sensor Networks 13(4), 241-253 (2013)

12. NE Huang, Z Shen, SR Long, MC Wu, HH Shih, Q Zheng, N-C Yen, CC Tung, $\mathrm{HH}$ Liu, The empirical mode decomposition and Hilbert spectrum for non-linear and non-stationary time series analysis. Proc. R. Soc. London A 454(1971), 903-995 (1998)

13. C Cai, W Liu, JS Fu, L Lilong, Empirical mode decomposition of micro-Doppler signature, in IEEE International Radar Conference, ed. by (Arlington, VA, USA). 9-12 May, 2005, pp 895-899

doi:10.1186/1687-6180-2014-75

Cite this article as: $\mathrm{Xu}$ and Liu: Vital sign sensing method based on EMD in terahertz band. EURASIP Journal on Advances in Signal Processing 2014 2014:75.

\section{Submit your manuscript to a SpringerOpen ${ }^{\circ}$ journal and benefit from:}

- Convenient online submission

- Rigorous peer review

- Immediate publication on acceptance

- Open access: articles freely available online

- High visibility within the field

- Retaining the copyright to your article

Submit your next manuscript at springeropen.com 